\title{
Systemic Treatment of Gastroesophageal Cancer during SARS-CoV2
}

\section{Background}

The SARS-CoV2 pandemic has its implications on the science and art of oncology. The morbidity and mortality associated with SARS-CoV2 are much higher compared to the general population. ${ }^{[1]}$ Data regarding the toxicity of anticancer therapies and their long-term impact are emerging. We have been pushed to a point where we have to weigh the risk-benefit ratio about a particular treatment regimen. To complicate the issue, the world has gone into significant travel restrictions both at the global and local levels. With massive lockdowns affecting multiple countries, there are restrictions on travel at the domestic level and local transport facilities are not available. Hospitals are overburdened with SARS-CoV2 patients, and triaging has taken a front seat in high-volume centers. In a situation like this, many governing bodies have come up with guidelines that cater to the needs and Do's and Don'ts for cancer patients. Here, we apply our collective wisdom and formulate guidelines for treatment recommendations of gastroesophageal cancer that might be applicable for our country where there is a possibility of SARS-CoV2 cases increasing with time.

Stomach cancer ranks as the $5^{\text {th }}$ most common cancer, followed by esophageal cancer in India. ${ }^{[2]}$ India has an age-standardized incidence rate of 5.0/100,000 population for the stomach and 4.5/100,000 population for the esophagus. This translates into approximately 51,000 deaths for the stomach and 46,000 for the esophagus every year. ${ }^{[2]}$ Esophageal cancer with squamous cell carcinoma (SCC) histology, mostly involving the lower and mid-esophagus, is mostly treated with a combination of neoadjuvant treatment (either chemotherapy or chemoradiotherapy) followed by surgery if resectable. ${ }^{[3]}$ Majority of them present in locally advanced or metastatic stages. Locally advanced stomach cancer is treated differently in different continents. The common approach has been upfront surgery followed by adjuvant chemoradiotherapy, ${ }^{[4]}$ perioperative chemotherapy like the MAGIC and the FLOT regimens, ${ }^{[5,6]}$ as well as adjuvant chemotherapy alone or chemoradiotherapy. ${ }^{[7,8]}$ All three approaches are practiced in India. None of these strategies come without adverse events. Grade 3 or 4 adverse events were reported in 279 of 496 patients $(56 \%)$ in the chemotherapy and surgery group in CLASSIC trial, ${ }^{[8]}$ and for ECF/ECX and FLOT regimens, the rate of hospitalization was $26 \%{ }^{[6]}$ Keeping in mind the resource constraints in the present situation, treatment de-escalation is the need of the hour.

Esophageal Cancer Involving the Upper and Middle Esophagus

Cervical esophageal cancer is primarily treated with protocols similar to head-and-neck unresectable tumors with concurrent chemoradiotherapy. Since the patients are usually symptomatic, withholding treatment may not be the best option. In this case, either single modality therapy like definitive radiotherapy (RT) may be more logical. In a study comparing RT with surgery, both have similar outcomes. There was no statistically significant difference in the progression-free and overall survival. ${ }^{[9]}$ In case of fit patients, combining chemotherapy with RT may be reasonable. However, in such a situation, it is better to avoid the toxic doublet regimen of cisplatin-5-fluorouracil (5FU), which is commonly used, and replace it with taxanecarboplatin, without compromising the outcomes. ${ }^{[10]}$

For the mid-esophagus, neoadjuvant chemotherapy (NACT) and chemoradiotherapy both are acceptable options. However, keeping toxicity in mind, giving NACT may be a better approach due to a more acceptable toxicity profile. In a meta-analysis comparing the two, 11 of 225 patients (4.89\%) suffered perioperative mortality in the NACT group, while 20 of 230 patients $(8.70 \%)$ suffered perioperative mortality in the neoadjuvant chemoradiotherapy group. ${ }^{[11]}$ Regimens best suited for concurrent would be either or taxane platinum combination in young for individuals. ${ }^{[12]}$

\section{For Gastroesophageal Junction and Stomach Cancer}

Historically, the MAGIC regimen was the standard of care for such tumors. This was recently replaced by the FLOT protocol (which is more commonly used in Europe), with better outcomes. However, there are intrinsic problems associated with both the protocols. The classic ECF regimen involved 21-day continuous infusion of 5FU, which is difficult to achieve in resource-constrained settings and not routinely done. An alternative to the ECF regimen is ECX where $5 \mathrm{FU}$ is replaced with capecitabine. ${ }^{[13]} \mathrm{In}$ the setting of SARS-CoV2, none of the above regimens are safe as far as toxicity and ease of administration is concerned. An alternative solution would be to use a safer regimen of taxane-platinum combination. For large bulky tumors where a better response rate is beneficial, RT may be combined with taxane-platinum. ${ }^{[12]}$

For patients who undergo upfront surgery, the standard treatment is adjuvant chemotherapy. In SARS-CoV2 state, it might be preferable to opt for single-agent capecitabine, as this can be taken at home, and blood reports may be evaluated through telemedicine. In patients who have pT1N1 disease, there is no benefit of adjuvant chemotherapy, and hence, it may be withheld. ${ }^{[14]}$

For Metastatic Esophageal, Gastroesophageal Junction, and Gastric Cancer

The most important factor deciding the treatment of metastatic disease is the baseline performance status. Since 
the intent is palliative, systemic chemotherapy should be withheld in cases with borderline low-performance status, with a lower threshold for palliative care. The main intent of therapy should be to provide relief of distressing symptoms.

For metastatic esophageal SCC, the most common regimen utilized is cisplatin-5FU. However, this may be modified to either single-agent platinum, preferable carboplatin, or a single-agent taxane. A weekly regimen is also an option, especially if the patient stays close to the hospital, as it leads to lesser degrees of hematological toxicities. For adenocarcinoma, ECX has been a standard regimen. However, it is better to avoid the triplet regimen during SARS-CoV2 and prescribe a safer doublet regimen like CAPOX or CAP-CIS. For ECOG PS 2, oral capecitabine, and for ECOG PS 3 and 4, the best supportive care option should be emphasized. For the Asian population, the doublet regimen is as good as a triplet based on meta-analysis. ${ }^{[15]}$ For elderly, frail individuals, either single-agent capecitabine or weekly paclitaxel is recommended. Toxic regimens such as DCF or mDCF should be avoided due to higher toxicities. Immunotherapy at the times of SARS-CoV2 does not have set guidelines. There are two concerns with immunotherapy and SARS-CoV2 infection. First, immunotherapy can cause interstitial pneumonitis, which might complicate the clinical picture of SARS-CoV2 infection, which is also known to manifest similarly. Second, immunotherapy can cause a cytokine storm, which may be detrimental in a SARS-CoV2 patient, since it can lead to further organ dysfunction. Hence, immunotherapy may be postponed till the crisis is over. Guidelines do not clearly support or refute the use of immune agents in SARS-CoV2 pandemic. Fortunately, the role of immunotherapy in the esophageal and gastroesophageal junction has not been promising, limiting the scope of its usage. The use of targeted agents like ramucirumab may be considered since they do not increase the toxicities significantly compared to single-agent taxanes. The use of single-agent ramucirumab is also acceptable if we really wish to avoid cytotoxic chemotherapy. ${ }^{[16]}$

To summarize, the overall picture of oncology and SARS-CoV2 is emerging. We do understand that it is difficult and sometimes unethical and humanely not possible to run randomized trials in such pandemics, and the only way to find the impact and outcome is through observational studies. Till we have very well-defined protocols, it is desirable to stick to regimens with lesser toxicity and put more emphasis on supportive and palliative care in poor outcome cancers like the esophagus and stomach.

$$
\begin{array}{r}
\text { Joydeep Ghosh'1, Sandip Ganguly', } \\
\text { Bivas Biswas }{ }^{1} \text {, Deepak Dabkara1, } \\
\text { Priyanka Srivastava², Amol Patel }{ }^{3}, \\
\text { Atul Batra4, Prashant Mehta }
\end{array}
$$

${ }^{\prime}$ Department of Medical Oncology, Tata Medical Center, Kolkata, West Bengal, India, ${ }^{2}$ Department of Medical Oncology, Shree Krishna Hospital and Medical Research Centre, Karamsad, Gujarat, India, ${ }^{3}$ Department of Medical Oncology, Malignant Diseases Treatment Centre, Army Hospital Research and Referral, New Delhi, India, ${ }^{4}$ Department of Medical Oncology, AIIMS, New Delhi, India, ${ }^{5}$ Department of Medical Oncology, Asian Institute of Medical Sciences, Faridabad, Haryana, India

Address for correspondence: Dr. Joydeep Ghosh, Department of Medical Oncology, Tata Medical Center, Kolkata, West Bengal, India. E-mail:dr.joydeep.ghosh@gmail.com

Submitted: $31-\mathrm{Mar}-2020$ Revised: 09-Apr-2020 Accepted: 17-Apr-2020 Published: 08-May-2020

\section{References}

1. Liang W, Guan W, Chen R, Wang W, Li J, Xu K, et al. Cancer patients in SARS-CoV-2 infection: A nationwide analysis in China. Lancet Oncol 2020;21:335-7.

2. Available from: https:/gco.iarc.fr/today/data/factsheets/ populations/356-india-fact-sheets.pdf. [Last accessed on 2020 Mar 31].

3. Gebski V, Burmeister B, Smithers BM, Foo K, Zalcberg J, Simes J, et al. Survival benefits from neoadjuvant chemoradiotherapy or chemotherapy in oesophageal carcinoma: A meta-analysis. Lancet Oncol 2007;8:226-34.

4. Macdonald JS, Smalley SR, Benedetti J, Hundahl SA, Estes NC, Stemmermann GN, et al. Chemoradiotherapy after surgery compared with surgery alone for adenocarcinoma of the stomach or gastroesophageal junction. N Engl J Med 2001;345:725-30

5. Cunningham D, Allum WH, Stenning SP, Thompson JN, Van de Velde CJ, Nicolson M, et al. Perioperative chemotherapy versus surgery alone for resectable gastroesophageal cancer. N Engl J Med 2006;355:11-20.

6. Al-Batran SE, Homann N, Pauligk C, Goetze TO, Meiler J, Kasper S, et al. Perioperative chemotherapy with fluorouracil plus leucovorin, oxaliplatin, and docetaxel versus fluorouracil or capecitabine plus cisplatin and epirubicin for locally advanced, resectable gastric or gastro-oesophageal junction adenocarcinoma (FLOT4): A randomised, phase 2/3 trial. Lancet 2019;393:1948-57.

7. Lee J, Lim DH, Kim S, Park SH, Park JO, Park YS, et al. Phase III trial comparing capecitabine plus cisplatin versus capecitabine plus cisplatin with concurrent capecitabine radiotherapy in completely resected gastric cancer with D2 lymph node dissection: The ARTIST trial. J Clin Oncol 2012;30:268-73.

8. Bang YJ, Kim YW, Yang HK, Chung HC, Park YK, Lee KH, et al. Adjuvant capecitabine and oxaliplatin for gastric cancer after D2 gastrectomy (CLASSIC): A phase 3 open-label, randomised controlled trial. Lancet 2012;379:315-21.

9. Cao CN, Luo JW, Gao L, Xu GZ, Yi JL, Huang XD, et al. Primary radiotherapy compared with primary surgery in cervical esophageal cancer. JAMA Otolaryngol Head Neck Surg 2014;140:918-26.

10. $\mathrm{Hu} \mathrm{G}$, Wang $\mathrm{Z}$, Wang $\mathrm{Y}$, Zhang Q, Tang $\mathrm{N}$, Guo $\mathrm{J}$, et al. Comparison of cisplatinum/paclitaxel with cisplatinum/5-fluorouracil as first-line therapy for nonsurgical locally advanced esophageal squamous cell carcinoma patients. Drug Des Devel Ther 2016;10:2129-36.

11. Zhao X, Ren Y, Hu Y, Cui N, Wang X, Cui Y. Neoadjuvant 
chemotherapy versus neoadjuvant chemoradiotherapy for cancer of the esophagus or the gastroesophageal junction: A meta-analysis based on clinical trials. PLoS One 2018;13:e0202185.

12. Noronha V, Prabhash K, Joshi A, Patil VM, Talole S, Nakti D, et al. Clinical outcome in definitive concurrent chemoradiation with weekly paclitaxel and carboplatin for locally advanced esophageal and junctional cancer. Oncol Res 2016;23:183-95.

13. Cunningham D, Starling N, Rao S, Iveson T, Nicolson M, Coxon F, et al. Capecitabine and oxaliplatin for advanced esophagogastric cancer. N Engl J Med 2008;358:36-46.

14. Shin HB, An JY, Lee SH, Choi YY, Kim JW, Sohn SS, et al. Is adjuvant chemotherapy necessary in pT1N1 gastric cancer? BMC Cancer 2017;17:287.

15. Guo X, Zhao F, Ma X, Shen G, Ren D, Zheng F, et al. A comparison between triplet and doublet chemotherapy in improving the survival of patients with advanced gastric cancer: a systematic review and meta-analysis. BMC Cancer 2019;19:1125.

16. Fuchs CS, Tomasek J, Yong CJ, Dumitru F, Passalacqua R, Goswami C, et al. Ramucirumab monotherapy for previously treated advanced gastric or gastro-oesophageal junction adenocarcinoma (REGARD): An international, randomised, multicentre, placebo-controlled, phase 3 trial. Lancet 2014;383:31-9.

This is an open access journal, and articles are distributed under the terms of the Creative Commons Attribution-NonCommercial-ShareAlike 4.0 License, which allows others to remix, tweak, and build upon the work non-commercially, as long as appropriate credit is given and the new creations are licensed under the identical terms.

For reprints contact:WKHLRPMedknow_reprints@wolterskluwer.com

\begin{tabular}{|l|l|}
\hline \multicolumn{2}{|c|}{ Access this article online } \\
\hline Quick Response Code: & Website: \\
& www.ijmpo.org \\
\cline { 2 - 2 } & DOI: \\
\hline
\end{tabular}

How to cite this article: Ghosh J, Ganguly S, Biswas B, Dabkara D, Srivastava $P$, Patel $A$, et al. Systemic treatment of gastroesophageal cancer during SARS-CoV2. Indian J Med Paediatr Oncol 2020;41:141-3. 